\title{
UNIFORM APPROXIMATION AND MAXIMAL IDEAL SPACES
}

\author{
JOHN WERMER ${ }^{1}$
}

Let $X$ be a compact set in the $z$-plane. We are interested in two function spaces associated with $X$ :

$C(X)=$ space of all continuous complex-valued functions on $X$.

$P(X)=$ space of all uniform limits of polynomials on $X$.

Thus a function $f$ on $X$ lies in $P(X)$ if there exists a sequence $\left\{P_{n}\right\}$ of polynomials converging to $f$ uniformly on $X$.

Clearly $P(X)$ is part of $C(X)$.

Question I. When is $P(X)=C(X)$, i.e. every continuous function approximable by polynomials?

Question II. If $P(X) \neq C(X)$, how can we characterize those functions on $X$ which lie in $P(X)$ ?

The first man to pay any attention to these problems I believe was Weierstrass who in 1885 showed $P(X)=C(X)$ when $X$ is the unit interval on the real axis. If instead of the unit interval we consider an arbitrary Jordan arc (homeomorph of the unit interval), the problem is much harder. Walsh [1] proved

Theorem. For every Jordan arc $J$ in the plane, $P(J)=C(J)$.

Instead of a Jordan arc, consider now a simple closed Jordan curve $\Gamma$. It is easy to see that now $P(\Gamma) \neq C(\Gamma)$. The reason is this: suppose $f$ is in $P(\Gamma)$. Then there is a sequence $\left\{P_{n}\right\}$ of polynomials tending to $f$ uniformly on $\Gamma$. Hence $\left|P_{n}-P_{m}\right|$ tends to 0 uniformly on $\Gamma$. If $z$ lies inside $\Gamma$,

$$
\left|P_{n}(z)-P_{m}(z)\right| \leqq \max _{\Gamma}\left|P_{n}-P_{m}\right|,
$$

by the maximum principle. Hence $P_{n}-P_{m}$ approaches 0 uniformly on $W$, the interior of $\Gamma$, whence $P_{n}$ tends to a limit uniformly on $W$; call it $F$. Clearly $F$ is analytic on $W$ and has $f$ as boundary values on $\Gamma$. Thus we have:

If $f$ is in $P(\Gamma)$, then $f$ is the boundary function of an analytic function. This rules out many $f$ 's in $C(\Gamma)$, e.g. any real-valued nonconstant $f$. This raises the question: Does $P(\Gamma)$ consist of all $f$ in $C(\Gamma)$ which are boundary function of functions analytic in $W$ ? Walsh [2] in 1926 showed

An address delivered before the New York meeting of the Society on February 25, 1961 by invitation of the Committee to Select Hour Speakers for Eastern Sectional Meetings; received by the editors March 30, 1962.

${ }^{1}$ Fellow of the Alfred P. Sloan Foundation. 
THEOREM. $P(\Gamma)$ consists of exactly those $f$ in $C(\Gamma)$ which admit an analytic continuation to the inside.

The preceding statement about arcs is a corollary. Consider now an arbitrary compact plane set $X$. What properties of $X$ are necessary in order that we can have $P(X)=C(X)$ ?

(1) $X$ has no interior (for each $f$ in $P(X)$ is analytic at interior points).

(2) The complement of $X$ is connected (for otherwise the complement has a bounded component, and then the same reasoning we just gave for a closed curve shows $P(X) \neq C(X))$.

In 1934 Lavrent'ev proved in [3]

Theorem. (1) and (2) are necessary and sufficient for $P(X)=C(X)$.

This settles Question I. What happens if we allow interior? Clearly, $P(X)$ contains only functions analytic on the interior of $X$. In 1951 Mergelyan [4] showed

THEOREM. If $X$ has connected complement, $P(X)$ consists of all $f$ in $C(X)$ which are analytic on the interior of $X$.

Mergelyan's result has all the other theorems as special cases. It settles Question II, essentially. After this result, it was natural that men should turn their eyes toward space, the space of $n$ complex variables, $C^{n}$.

Let $X$ be a compact subset of $C^{n}$ and define $P(X)$ and $C(X)$ as before, only now with "polynomial" meaning polynomial in $z_{1}, \cdots, z_{n}$, rather than in $z$. Again we ask Questions I and II for these new sets and our hope is to find some analogue of Mergelyan's theorem for $C^{n}$. This goal is up to now very far from having been reached. However, a number of interesting things have been found out in the last 10 years and a few of these I want to discuss.

$P(X)$ has two structures: it is an algebra over the complex numbers under pointwise addition and multiplication of functions, and it is a Banach space under the norm $\|f\|=\max _{x}|f|$. Since $\|f \cdot g\| \leqq\|f\| \cdot\|g\|$, $P(X)$ is thus a Banach algebra, and it is clearly commutative, has a unit, and is semi-simple. An important object attached to a commutative Banach algebra is its space of maximal ideals. How can we identify that space for an algebra $P(X)$ ? The general theory of Banach algebras sets up a one-to-one correspondence between maximal ideals $M$ and linear functionals $m(m \neq 0)$ on the algebra which are multiplicative $(m(x y)=m(x) m(y))$, such that $M$ is the kernel of $m$. Also $m$ has norm 1 as linear functional. 
Let $m$ be a multiplicative linear functional on $P(X)$. The coordinate functions $z_{1}, \cdots, z_{n}$ generate $P(X)$ as Banach algebra, so $m$ is determined by the $n$-tuple of numbers $\left(m\left(z_{1}\right), \cdots, m\left(z_{n}\right)\right)$. This $n$ tuple is a certain point $\phi(m)$ in $C^{n}$. This point has the property

$$
|P(\phi(m))| \leqq \max _{\boldsymbol{X}}|P|, \quad \text { for all polynomials } P \text {. }
$$

This is true because $P(\phi(m))=m\left(P\left(z_{1}, \cdots, z_{n}\right)\right)$ since $m$ is linear and multiplicative, and $\|m\|=1$.

Definition. The hull of $X$ is the set $h(X)$ of all points $x_{0}$ in $C^{n}$ such that for all polynomials $P$ we have $\left|P\left(x_{0}\right)\right| \leqq \max _{X}|P|$.

The map $\phi$ just defined attaches to each multiplicative linear functional $m$ on $P(X)$ a point of the hull of $X$. It is easy to see that $\phi$ is one-one and onto so that we can identify the maximal ideal space (space of $m$ 's) with the hull.

For $X$ in the complex plane, $h(X)$ is very easy to describe: it is the union of $X$ and its bounded complementary components, i.e. it is obtained by filling in the holes in $X$. As an example in $C^{2}$, consider the set $X$ of all $(z, w),|z|=1,|w|=1$. Thus $X$ is a torus. Here $h(X)$ is the closed bicylinder $|z| \leqq 1,|w| \leqq 1$. Already this example shows that "filling in holes" is much subtler in $C^{2}$ than in the plane.

For an arbitrary compact $X$ in $C^{n}$ it is clear from the definition that $h(X)$ is compact and contains $X$. What else can we say about it? By a Weil polyhedron in $C^{n}$ we mean a set $W$ where

$$
W=\left\{x \text { in } C^{n}|| P_{i}(x) \mid<1, i=1, \cdots, k\right\},
$$

the $P_{\boldsymbol{i}}$ being polynomials. A. Weil proved in the Thirties that if $F$ is any analytic function on such a set $W$, then $F$ is uniformly approximable on compact subsets of $W$ by polynomials. Silov [5] showed:

THEOREM. Let $X$ be a compact set in $C^{n}$ and $F$ a function defined and analytic in some neighborhood of $h(X)$. Then $F$ (restricted to $X)$ is in $P(X)$.

To prove this, we observe that if $x_{0}$ is not in $h(X)$, then by definition of hull there exists a polynomial $Q$ with $\left|Q\left(x_{0}\right)\right|=1,|Q|<1$ on $h(X)$. From this one easily gets the existence of a Weil polyhedron containing $h(X)$ inside every neighborhood of $h(X)$, in particular inside the neighborhood where $F$ is given. Applying Weil's result, we get that $F$ is in $P(X)$.

Corollary (Silov [5]). If $X$ is connected, then $h(X)$ is connected.

Further topological information on hulls was recently given by A. Browder [6], who makes use of a result of Serre [7]. 
TheOREM (BRowder). If $X$ is a compact set in $C^{n}$, then the jth Cech cohomology group of $h(X)$ with complex coefficients, $H^{i}(h(X), C)$, vanishes for $j \geqq n$.

Let us call a set $X$ "polynomially convex" if it coincides with its hull, i.e. $h(X)=X$. The last theorem has the interesting consequence that if $X$ is topologically a 2 -sphere (or any compact orientable 2 manifold) in $C^{2}$, then $X$ cannot be polynomially convex, for then the second cohomology group of $X$ would be 0 . Of course the analogous statement holds for other $C^{n}$.

A simple sufficient condition for polynomial convexity is

Theorem. If $P(X)=C(X)$, then $X$ is polynomially convex.

The reason for this is that $h(X)$ is the maximal ideal space of $P(X)$ as we saw earlier, and the maximal ideal space of $C(X)$ is easily seen to be $X$ itself. As a consequence we have:

Corollary. If $X$ lies in the real subspace of $C^{n}$, i.e. the subspace of points all of whose coordinates are real, then $X$ is polynomially convex.

For $P(X)$ is here generated by functions real on $X$, and so the Stone-Weierstrass theorem yields $P(X)=C(X)$. This Corollary shows that the lower dimensional cohomology groups $(j<n)$ of a polynomially convex set are highly arbitrary.

I next want to discuss what happens to Walsh's theorems about curves and arcs when these lie in $C^{n}, n>1$.

Let $\Gamma$ be a simple closed curve in $C^{n}, n>1$. It is quite possible that $\Gamma$ is polynomially convex, e.g. if $\Gamma$ lies in the real subspace. On the other hand, assume that $\Gamma$ lies on an analytic variety $\Sigma$ of complex dimension 1 and that $\Gamma$ bounds a compact piece of $\Sigma$. By the maximum principle on $\Sigma$, then, that compact piece will lie in $h(\Gamma)$. In the converse direction one can say the following, proved by the author in [8]: Call $\Gamma$ an analytic curve if it can be represented in the form $z_{i}=\phi_{i}(t), i=1, \cdots, n$, where $t$ ranges over the circle $|t|=1$, where the $\phi_{i}$ are analytic on $|t|=1$ and $\phi_{1}^{\prime}(t) \neq 0$ on $|t|=1$.

THEOREM. Let $\Gamma$ be an analytic curve in $C^{n}$ which is not polynomially convex. Then $\Gamma$ lies on an analytic variety of complex dimension 1 and bounds on this variety a compact piece. That compact piece is then the hull of $\Gamma$.

Let next $J$ be a Jordan arc in $C^{n}$. Call it "analytic" if it can be parametrized $z_{i}=\phi_{i}(t), 0 \leqq t \leqq 1$, with the $\phi_{i}$ analytic on $[0,1]$ and $\phi_{1}^{\prime} \neq 0$. In [8] we showed that, as one would expect: 
THEOREM. Every analytic arc in $C^{n}$ is polynomially convex, and moreover $P(J)=C(J)$.

Both these theorems become false for arbitrary Jordan curves and arcs in $C^{n}$. Thus there exists a simple closed curve whose hull has (real) dimension $\geqq 4$. Also the author [9] and Rudin [10] constructed Jordan arcs, in $C^{3}$ and $C^{2}$ respectively, which are not polynomially convex. The construction in $C^{3}$ proceeds by exhibiting a 2 sphere in $C^{3}$ containing an arc such that the sphere minus the arc is an analytic variety. If $P$ is any polynomial, $|P|$ takes its maximum somewhere on the sphere. By the maximum principle for analytic varieties, it must take it on the arc. Hence the hull of the arc contains the sphere. On the other hand, the hypothesis of analyticity can be considerably weakened. (See Bishop [11]). The following interesting and apparently difficult questions are open:

If $J$ is a differentiable arc in $C^{n}$, must $P(J)=C(J)$ ? $^{2}$

If $J$ is a Jordan arc in $C^{n}$ which is polynomially convex, must $P(J)$ $=C(J)$ ?

As a partial generalization of Walsh's theorem on simple closed curves, one has the following theorem:

TheORem. Let $\Gamma$ be an analytic simple closed curve in $C^{n}$ which lies on a 1-complex-dimensional variety $\Sigma$ and bounds on $\Sigma$ a nonsingular compact piece $\Sigma_{0}$. Then $P(\Gamma)$ consists of exactly those $f$ in $C(\Gamma)$ which admit analytic extensions to $\Sigma_{0}-\Gamma$.

This is seen by combining results of the author in [8] and of Bishop [12]. Various related results on algebras of analytic functions on Riemann surfaces are given in Bishop $[11 ; 12]$ and Rossi [13].

We now leave curves and arcs and consider again a general compact set $X$. The functions in $P(X)$ admit a certain natural extension from $X$ to all of $h(X)$. To see this, let $f$ belong to $P(X)$ and let $\left\{P_{n}\right\}$ be a sequence of polynomials tending to $f$ on $X$. It then follows at once from the definition of $h(X)$ that $\left\{P_{n}\right\}$ converges uniformly on $h(X)$ to a continuous limit function $\hat{f}$, and that $\hat{f}$ depends only on $f$, and so provides the asserted extension of $f$ to $h(X)$. (The map: $f \rightarrow \hat{f}$ is in fact the Gelfand representation of $P(X)$ by continuous functions on its maximal ideal space.)

If $h(X)-X$ is an open set in $C^{n}$, as is for instance the case when $X$ is a simple closed curve in the plane, then each $\hat{f}$ is clearly an analytic

${ }^{2}$ Added in proof. Answered affirmatively by Bishop, as a consequence of results in [11]. 
function on $h(X)-X$. Is it true in the general case that the $\hat{f}$ have certain properties of analyticity on $h(X)-X$ ?

In this direction Rossi [14] has proved the following generalization of the local form of the classical Maximum Principle:

Theorem. Let $X$ be any compact set in $C^{n}$. Let $x_{0}$ be a point in $h(X)$ $-X, V$ a neighborhood of $x_{0}$ in $h(X)-X$ and $\partial V$ the boundary of $V$ in $h(X)$. Then if $f$ is any element of $P(X)$, we have

$$
\left|\hat{f}\left(x_{0}\right)\right| \leqq \max _{\partial V}|\hat{f}|
$$

The proof makes use of the result of Weil mentioned earlier and of the solution of the so-called Cousin Problem (existence of meromorphic functions with prescribed zeros and poles) for certain regions.

The question had been raised whether perhaps for every $X$, $h(X)-X$ contains pieces of analytic varieties. This has unfortunately been answered in the negative, by Stolzenberg in [19]. The question: for what geometrical reason does a given point outside a set $X$ lies in the hull of that set? has not been answered in a satisfactory way, and remains a tantalizing problem.

Let us finally return to the $z$-plane and consider another function space associated with a compact plane set $X$ :

$R(X)=$ space of uniform limits on $X$ of rational functions which are analytic on $X$.

The holes in the set $X$ play a quite different role for $R(X)$ than for $P(X)$. For if $z_{0}$ lies in such a hole, $\left(z-z_{0}\right)^{-1}$ belongs to $R(X)$, and so functions in $R(X)$ need not be analytic in the hole. As before, the condition: " $X$ has no interior" is necessary for $R(X)=C(X)$. It is however not sufficient, as the following example, given by Mergelyan in [16], shows: Let $S$ be the compact set obtained by removing from the closed unit disk $D$ an infinite family of disjoint open disks $D_{i}$ in such a way that the sum of the lengths of the boundaries of the $D_{i}$ converges, while $S=D-\cup_{i} D_{i}$ has no interior points. The set $S$ is known as a Swiss Cheese, for obvious reasons. On $C(S)$ one can now define the following bounded linear functional $L$, where $\gamma_{i}$ denotes the boundary of $D_{i}$ :

$$
L(f)=\int_{|z|=1} f(z) d z+\sum_{i=1}^{\infty} \int_{\gamma_{i}} f(z) d z,
$$

integrating in the positive direction over $|z|=1$ and in the negative direction over the $\gamma_{i}$. It now follows by Cauchy's theorem that if $g$ is any rational function whose poles lie in the complement of $S$, then 
$L(g)=0$. Hence $L$ annihilates $R(S)$. Clearly also $L$ does not annihilate $C(S)$, and so $R(S) \neq C(S)$ although $S$ is without interior.

Bishop and Hoffman have shown that one can map the set $S$ homeomorphically on a set $S^{\prime}$ in $C^{2}$ in such a way that the function space $R(S)$ goes over into the function space $P\left(S^{\prime}\right)$. Thus approximation by rational functions in one variable is here equivalent to polynomial approximation in two variables. A general result of this type is given by Rossi [15]. Swiss Cheese sets are the basis of numerous counterexamples in approximation theory; in particular, the abovementioned counterexample of Stolzenberg rests on such a set.

One answer to the question: when does $R(X)$ equal $C(X)$ ? is given in the following result of Bishop [17]:

THeOREm. Let $X$ be a compact plane set. The condition:

(*) For each $x_{0}$ in $X$, there exists $f$ in $R(X)$ with $\left|f\left(x_{0}\right)\right|>|f(y)|$ for all $y$ in $X-x_{0}$, is necessary and sufficient for $R(X)=C(X)$.

Various geometric conditions on a set $X$ are known assuring that $R(X)=C(X)$. For such results see Mergelyan [16]. A simple condition given in 1931 by Hartogs and Rosenthal [18] is the following:

Theorem. If $X$ has vanishing plane Lebesgue measure, then $R(X)$ $=C(X)$.

A necessary and sufficient geometric condition on $X$ does not seem to be known at the present.

\section{REFERENCES}

A more detailed and extensive article on the problems mentioned in this talk and on more general questions concerning algebras of continuous functions was written by the author in the Advances in Mathematics series of the Academic Press. It is titled Banach algebras and analytic functions and appears in Volume 1, Fascicle 1, 1961.

1. J. L. Walsh, Interpolation and approximation by rational functions in the complex domain, Amer. Math. Soc. Colloq. Publ. Vol. 20, Amer. Math. Soc., Providence, R. I., 1935.

2. - Über die Entwicklung einer analytischen Funktion nach Polynomen, Math. Ann. 96 (1926), 430-436, 437-450.

3. M. A. Lavrent'ev, Sur les fonctions d'une variable complexe représentable par des séries de polynomes, Hermann, Paris, 1936.

4. S. N. Mergelyan, On the representation of functions by series of polynomials on closed sets, Dokl. Akad. Nauk SSSR (N.S.) 78 (1951), 405-408-Amer. Math. Soc. Transl. 85 (1953).

5. G. E. Silov, On the decomposition of a commutative normed ring into a direct sum of ideals, Amer. Math. Soc. Transl. (2) 1 (1955).

6. A. Browder, Cohomology of maximal ideal spaces, Bull. Amer. Math. Soc. 67 (1961), 515-516. 
7. J.-P. Serre, Une propriêté topologique des domaines de Runge, Proc. Amer. Math. Soc. 6 (1955), 133-134.

8. J. Wermer, The hull of a curve in $C^{n}$, Ann. of Math. (2) 68 (1958), 550-561. This paper is dependent on the following three papers of the author:

Function rings and Riemann surfaces, Ann. of Math. (2) 67 (1958).

Rings of analytic functions, Ann. of Math. (2) 67 (1958).

Subalgebras of the algebra of all complex-valued continuous functions on the circle, Amer. J. Math. (2) 78 (1954).

9. - Polynomial approximation on an arc in $C^{3}$, Ann. of Math. 62 (1955), 269-270.

10. W. Rudin, Subalgebras of spaces of continuous functions, Proc. Amer. Math. Soc. 7 (1956), 825-830.

11. E. Bishop, Analyticity in certain Banach algebras, Trans. Amer. Math. Soc. 102 (1962), 507-544.

12. - Subalgebras of functions on a Riemann surface, Pacific J. Math. 8 (1958), 29-50.

13. H. Rossi, Algebras of holomorphic functions on one-dimensional varieties, Trans. Amer. Math. Soc. 100 (1961), 439-458. $1-11$.

14. - The local maximum modulus principle, Ann. of Math. (2) 72 (1960),

15. - Holomorphically convex sets in several complex variables, Ann. of Math.

(2) 74. (1961), 470-493.

16. S. N. Mergelyan, Uniform approximations to functions of a complex variable, Amer. Math. Soc. Transl. 101 (1954).

17. E. Bishop, A minimal boundary for function algebras, Pacific J. Math. 9 (1959), 629-642.

18. F. Hartogs and A. Rosenthal, Über Folgen analytischer Funktionen, Math. Ann. 104 (1931), 606-610.

19. G. Stolzenberg, Massachusetts Institute of Technology thesis, 1961.

Brown UnIVERsity

\section{RESEARCH PROBLEM}

33. E. W. Cheney and P. C. Curtis, Jr., Convex bodies.

Let $M$ denote a linear manifold (i.e. translate of a linear subspace) in $n$-dimensional real space. For each real $p>1$ there is a unique point $x_{p}=\left(\xi_{p 1}, \cdots, \xi_{p n}\right)$ on $M$ for which the norm $\left(\sum_{j=1}^{n}\left|\xi_{p j}\right|^{p}\right)^{1 / p}$ is a minimum. Prove or disprove the conjecture that $\lim _{p \rightarrow \infty} x_{p}$ exists in all cases. The conjecture has been established by simple arguments when $n \leqq 3$, when $M$ has dimension 1 or $n-1$, and when there exists a unique point $x_{0}=\left(\xi_{01}, \cdots, \xi_{0 n}\right)$ on $M$ for which $\max _{j}\left|\xi_{0 j}\right|$ is a minimum. (Received January 20, 1962.) 